\title{
La sparterie selon Jean-François Gavoty
}

D’un matériau « pauvre » à un produit de luxe

Plaiting According to Jean-François Gavoty. From a "Poor" Material to a Luxury Product

\section{Christiane Demeulenaere-Douyère}

\section{OpenEdition \\ Journals}

Édition électronique

URL : https://journals.openedition.org/tc/7939

DOI : $10.4000 /$ tc. 7939

ISSN : 1952-420X

Éditeur

Éditions de l'EHESS

\section{Édition imprimée}

Date de publication : 31 octobre 2016

Pagination : 180-193

ISBN : 9782713225291

ISSN : 0248-6016

Référence électronique

Christiane Demeulenaere-Douyère, «La sparterie selon Jean-François Gavoty », Techniques \& Culture [En ligne], 65-66 | 2016, mis en ligne le 31 octobre 2018, consulté le 29 septembre 2022. URL : http:// journals.openedition.org/tc/7939; DOI : https://doi.org/10.4000/tc.7939 


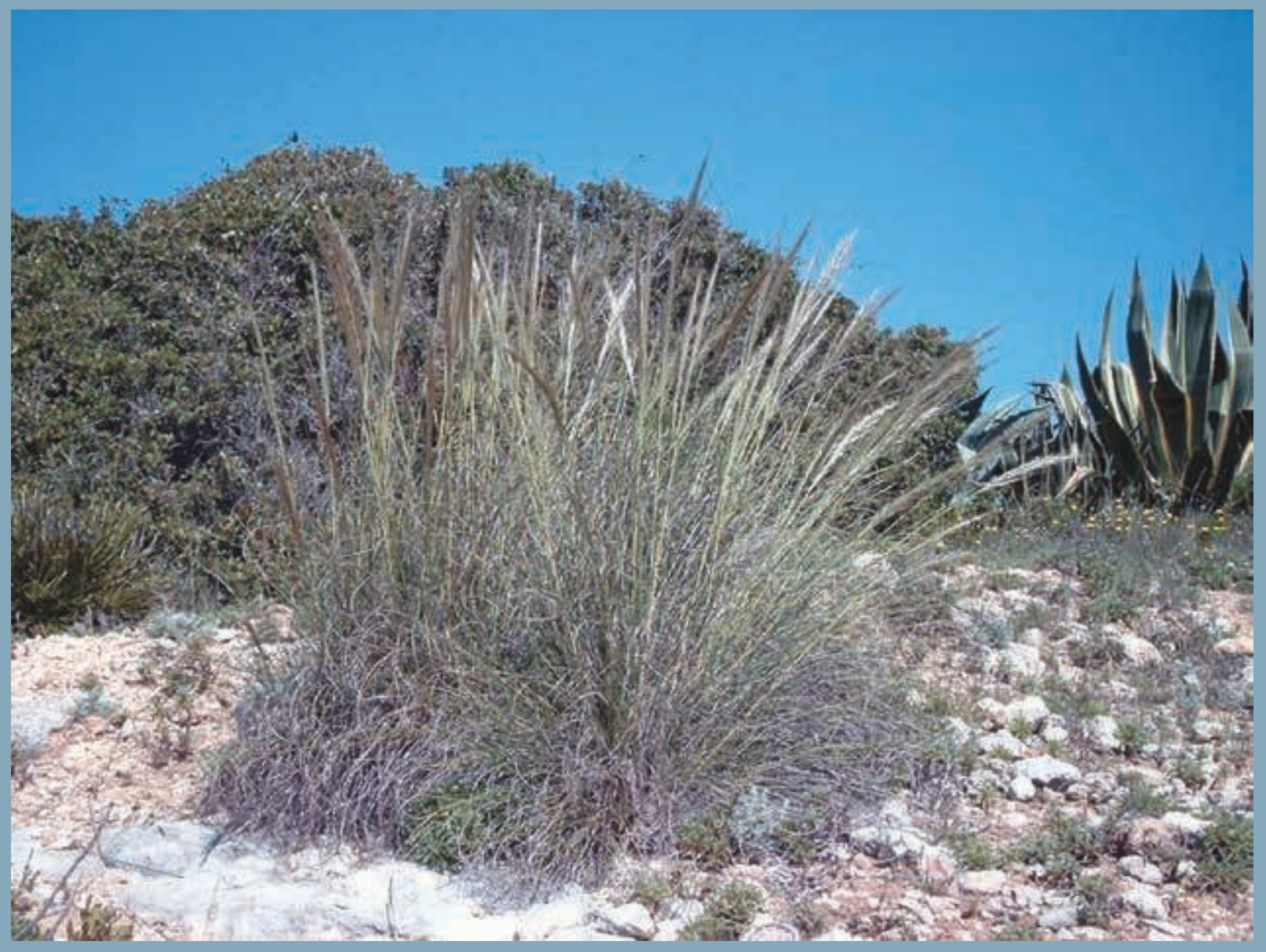




\section{La sparterie selon Jean-François Gavoty D’un matériau " pauvre " à un produit de luxe}

Si l'on en croit les médias, l'art d'«accommoder les restes» est une préoccupation majeure de notre siècle, de plus en plus contraint, nous dit-on, à rechercher des alternatives à une consommation excessive, non régulée, génératrice de trop nombreux «restes», dont la valorisation devient par ailleurs une obligation coûteuse. Mais, au-delà du strict aspect du recyclage des restes, on peut décentrer un peu la question posée dans ce numéro de TechniquesÉCulture en l'abordant sous l'angle d'une gestion raisonnée des matières premières, réservant à chacune l'usage le mieux approprié et le plus économiquement rentable. Il s'agit moins de recyclage à proprement parler que d'une alternative nouvelle apportée à un problème de matières premières.

D’autres époques se sont trouvées confrontées à la double question de la raréfaction de la matière première et d'une demande du marché plus exigeante; elles aussi ont essayé d'inventer des solutions. Ainsi, l'utilisation du spart, dont la promotion est soutenue par le cordier Gavoty à la fin du xviII siècle, s'inscrit dans le souci de réévaluer un matériau «pauvre», ou considéré comme tel, poussant naturellement, sans intervention humaine, mais méprisé pour sa faible valeur marchande et jusque-là abandonné aux usages populaires. Il s'agit d'une utilisation innovante d'un matériau "archaïque», laissé pour compte, pour en faire une matière première «riche», qui peut même déboucher, grâce au savoir-faire de l'entrepreneur et à l'engouement de l'opinion publique, sur des productions de luxe.

Dans la seconde moitié du XviII ${ }^{\text {e }}$ siècle, la production de chanvre est en recul sur le territoire français, même si sa culture reste implantée dans certains centres de production en Bretagne et dans le Perche. Pour ses approvisionnements, le royaume devient dépendant de l'étranger et doit, pour fournir les tissages et particulièrement la Marine royale qui a besoin de chanvre en qualité et en quantité suffisantes pour ses voiles et ses cordages, importer des chanvres bruts ou ouvragés d'Italie et d'Europe du Nord (Allemagne, Prusse, Hollande, villes hanséatiques, Russie) (Rougier de la Bergerie 1800). 
Objets divers en sparterie réalisés par le CETREP.

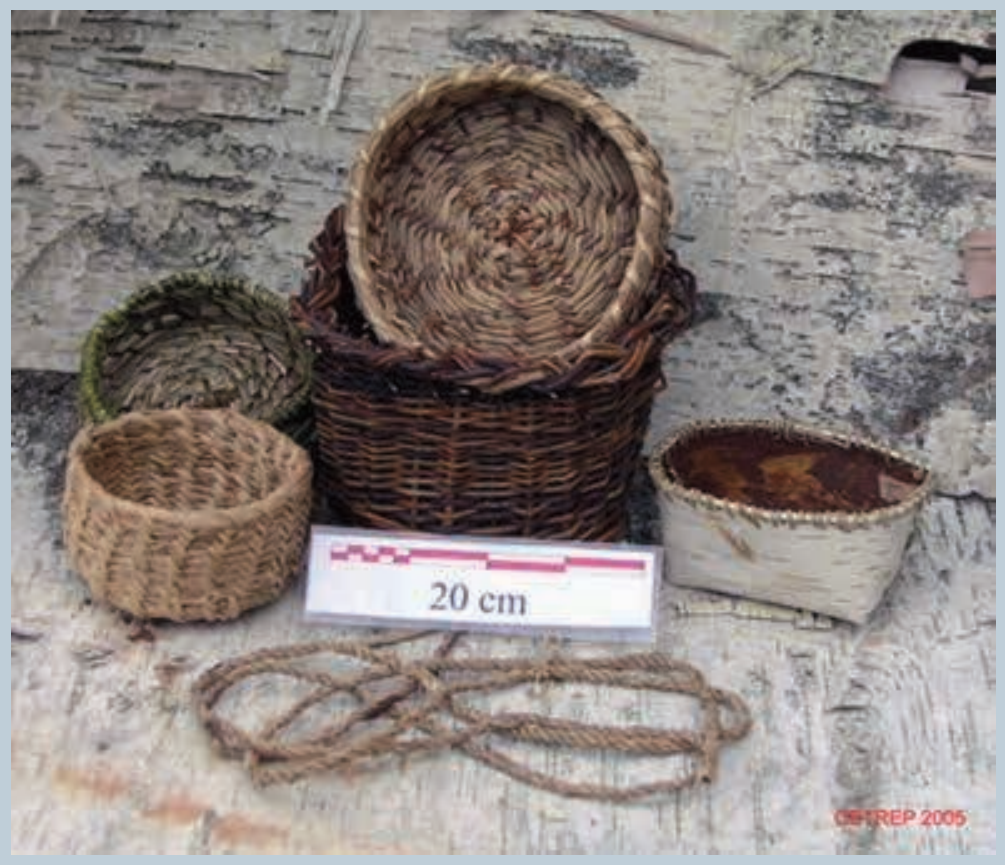

2. Objets en sparterie. Sparterie de Dammartin-en-Serve (78).

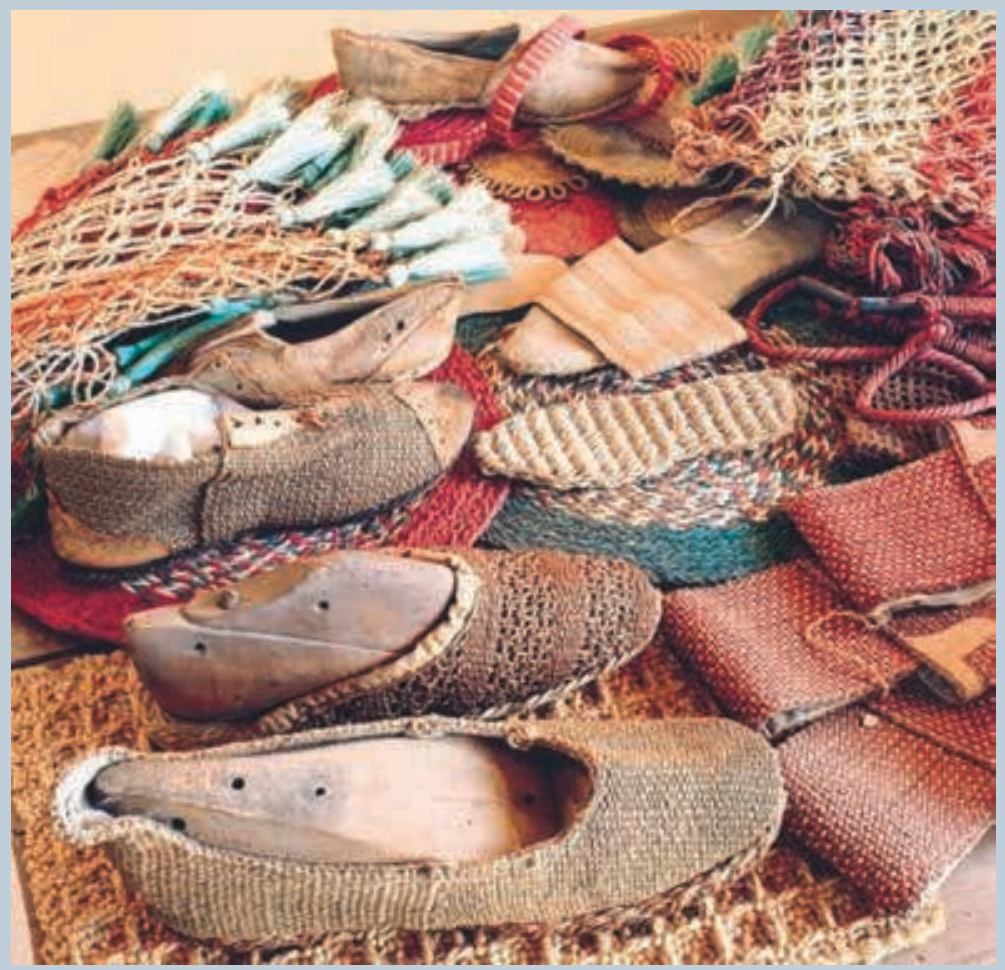


Le chanvre n'est pas utilisé seulement pour faire des toiles et des cordages pour la marine; on lui connaît une grande diversité d'utilisations, notamment dans l'industrie, par exemple dans les papeteries où il fournit les cordes sur lesquelles on met le papier à sécher. Il est aussi très utilisé pour des usages domestiques (il fournit des cordes, ficelles, nattes, cabas, tapis, etc.), en concurrence avec la paille que les Physiocrates préféreraient voir consacrée à d'autres usages, notamment agricoles comme l'engrais des terres ou encore la nourriture et la litière du bétail. L'importance stratégique que donne au chanvre son utilité pour la marine et la valeur économique de la paille pour l'agriculture et l'élevage vont pousser des manufacturiers à rechercher des substituts à ces matières premières.

\section{La redécouverte du spart en France}

Le spart est connu de longue date ${ }^{1}$ et a été décrit par Pline. Cette dénomination recouvre en fait deux plantes, le lygeum spartum (spart ou faux spart) et la stipa tenacissima (alfa). Dans les deux cas, il s'agit de graminées fourragères, de la famille des poacées (Poaceae), qui croissent naturellement, sans intervention humaine, sur des sols pauvres et arides, principalement autour de la Méditerranée (Afrique du Nord, Espagne), où elles peuvent occuper de vastes espaces ${ }^{2}$. Dans tout le pourtour du bassin méditerranéen, ces fibres sont traditionnellement utilisées pour des usages domestiques, industriels ${ }^{3}$ ou maritimes ${ }^{4}$ : au printemps, la feuille est tressée pour confectionner divers objets de sparterie (paniers, couffins, nattes, etc.) et les fibres tirées des feuilles peuvent, après avoir été filées, s'employer pour la fabrication de cordages. Le spart a la caractéristique d'être assez solide, mais surtout de bien résister à l'humidité et donc au pourrissement.

Il faut noter que la transformation du spart fait partie de ces techniques «archaïques», anciennement ancrées dans les usages vernaculaires, avec lesquelles on tend à renouer à la faveur de la crise de la fin du Xviıle siècle, face à l'épuisement de certaines ressources naturelles et à l'accroissement de la demande du marché5 (Baridon, Garric, Richaud 2016; DemeulenaereDouyère in Fleury et al. 2016).

Jean-François Gavoty, qui se fait appeler aussi Gavoty de Berthe, est un Provençal, né à Toulon (Var) en $1733^{6}$. Il monte à Paris en 1769, «pour s'y perfectionner, dit-il, dans la culture des sciences et des arts, et des trois règnes de la nature, dont il s'occupait depuis la plus tendre jeunesse» (Gavoty 1792). Il a alors 36 ans. On ne sait rien de ses activités antérieures, sinon qu'il appartient à une famille de négociants de Toulon ${ }^{7}$; certains auteurs affirment qu'il «a résidé longtemps en Espagne» (Lunier 1805).

En 1773 a lieu dans le royaume une grande arrestation de mendiants. Cet événement donne à Gavoty l'idée de mettre à profit cette main-d'œuvre pour, sous prétexte de l'« accoutumer au travail», introduire et développer en France une industrie du spart. Ce dessein lui est inspiré par son expérience personnelle: «Les provençaux l'importaient [...] et les galériens, le peuple et les gens de la campagne faisaient des tresses, des nattes, des cabas et des cordages d'un très bon usage.» (Gavoty op. cit.: 4). 


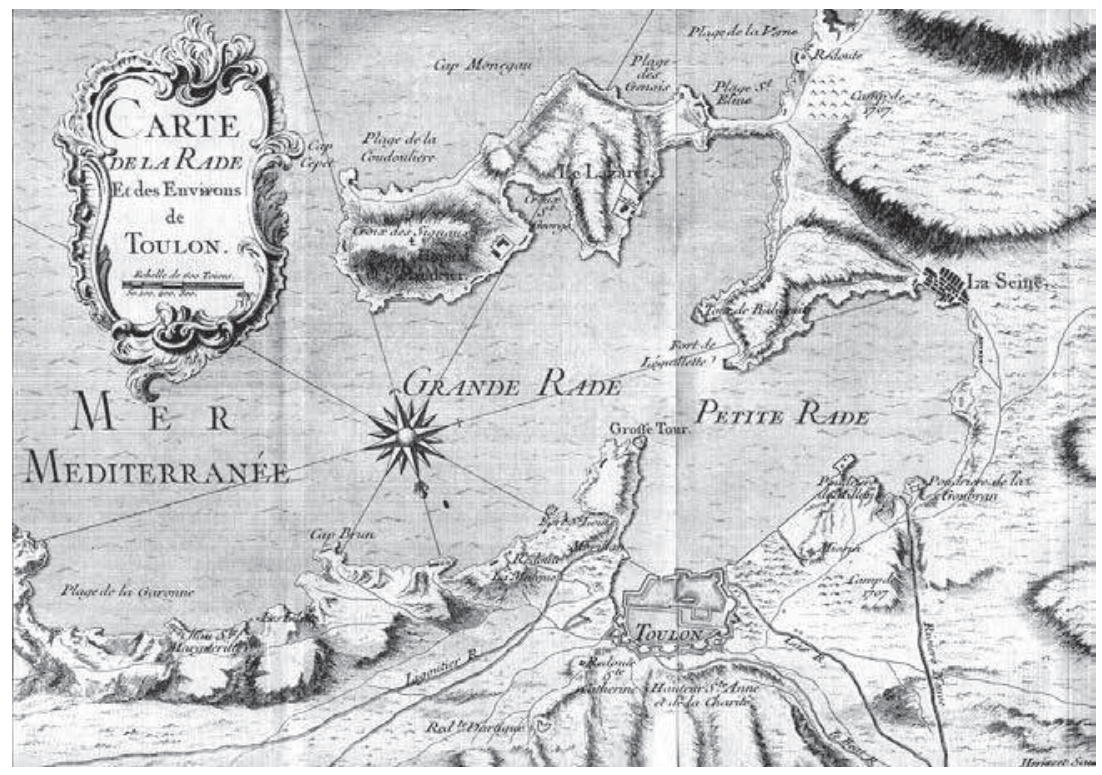

On peut s'interroger sur ce qui l'emporte chez lui, des motivations philanthropiques qu'il met volontiers en avant (il veut prévenir la mendicité en France), ou de l'opportunité d'obtenir à bon compte une main-d'œuvre peu ou pas rémunérée pour favoriser ses projets industriels. Il fait venir du spart d'Espagne, fait des essais avec des mendiants qu'il exerce au tressage et au filage de cette matière, et «bientôt, écrit-il, l'art de la corderie fut perfectionné».

\section{La création de la "Corderie nouvelle " de Popincourt}

Carte de la rade et des environs de Toulon Publiée par Bélidor dans L'architecture hydraulique, seconde partie, tome second, pl. Iv, 1753. (Coll. B. Cros).
Gavoty projette de créer à Paris un établissement de transformation du spart. Bénéficiant de protections bien placées - l'intendant de Bourges, Nicolas Dupré de Saint-Maur, et le directeur des Ponts et Chaussées, Trudaine de Montigny-, il négocie avec le gouvernement pour en obtenir l'autorisation, mais ne sollicite ni de privilège exclusif ni le titre de manufacture royale. Il met en avant une argumentation économique:

«Le haut prix du chanvre et des ouvrages qui en occasionnent la consommation, et celuy de la paille par l'employ qui s'en fait à Paris à des ouvrages qui en détournent l'application de la nourriture et litière des bestiaux et pour l'engrais des terres, luy ont fait imaginer de chercher des moyens pour laisser ces matières aux objets pour lesquels elles sont essentiellement propres $^{8}$.»

Il préconise de réserver l'usage du chanvre à la marine et celui de la paille à l'agriculture et à l'élevage, ce qui ne peut que remporter l'adhésion des Physiocrates, tout comme d'ailleurs son projet d'exploiter des terres arides et improductives pour cultiver le spart ${ }^{9}$. Par ailleurs, la transformation du spart, bien préparé, permettra de livrer au commerce des marchandises équivalentes en qualité ou même plus durables que celles obtenues avec le chanvre et la paille.

Son projet est soumis à l'examen de l'Académie royale des sciences ${ }^{10}$ qui est consultée sur toutes les questions touchant à l'innovation technique. Aux yeux des académiciens, le spart présente un atout considérable: dans les papeteries, les cordes de spart, même moins bien préparées que celles de Gavoty, ont l'avantage sur celles faites de chanvre de s'imbiber difficilement de l'humidité des feuilles de papier qu'on étend dessus et donc de pourrir moins vite; de plus, ne conservant pas l'humidité, elles préservent le pli des feuilles de papier de la formation de 
rides. Ils font des expériences sur la résistance du spart et constatent «qu'une corde de 3 pouces de circonférence et pesant 1 livre 11 onces par toise, a soutenu le poids de 2160 livres et n'a cassé qu'à l'endroit où elle embrassait la poulie d'une grue qu'en conséquence d'un tour forcé qu'on lui a fait éprouver».

Ils concluent que «le jonc d'Espagne est de nature à pouvoir suppléer le chanvre dans plusieurs circonstances et à faire mieux que la paille et les écorces».

L'Académie des sciences soutient donc la demande de Gavoty, d'autant que celui-ci propose d'«introduire en même temps dans quelques terrains vagues des provinces méridionales la culture du jonc d'Espagne».

Finalement, le 3 octobre $1775^{11}$, Gavoty obtient du Conseil du roi l'autorisation de faire fabriquer les ouvrages de spart à Paris et dans les environs, de vendre et faire vendre à Paris et dans les environs des ouvrages de spart fabriqués dans sa manufacture ou importés par lui d'Espagne, et de cultiver le spart «dans les terrains vagues des provinces méridionales». C'est un véritable complexe industriel et commercial qui reçoit le soutien royal: production de la matière première, transformation de cette matière première et débit des produits manufacturés, produits en France ou importés.

Et, effectivement, dès l'année suivante, en 1776, il ouvre une manufacture de sparterie à Paris, rue Popincourt, dans ce faubourg Saint-Antoine qui, situé à l'écart des contraintes corporatistes, a été une extraordinaire pépinière d'innovations techniques (Thillay 2002). Pour installer son industrie qui nécessite beaucoup d'espace, peut-être bénéficie-il des bâtiments du couvent des Annonciades de Popincourt, alors en cours de liquidation (Garin 1910).

Sur l'organisation interne de la manufacture et sur son outillage, on manque, faute de documentation, d'éléments précis. Dans un opuscule publié bien plus tardivement (en 1810) ${ }^{12}$, Gavoty décrit des éléments qu'il estime indispensables à la bonne fabrication des câbles de chanvre, qu'il prétend avoir réunis dans «un établissement [...] que je formai en 1776, rue de Popincourt, faubourg St-Antoine, [...] connu sous le nom de Manufacture de Sparterie»:

« $1^{\circ}$ un long terrain; $2^{\circ}$ des ateliers de peignerie, de filerie et de comettage; $3^{\circ}$ des outils et des ustensiles en quantité; $4^{\circ}$ des chanvres rudes et élastiques, des chanvres doux et flexibles, et des filasses provenant d'autres végétaux; $5^{\circ}$ un laboratoire de chimie pour connaître la constitution des plantes de plusieurs familles, d'après la décomposition de leurs filasses; $6^{\circ}$ Des ouvriers, pour les instruire des règles du peignage, du filage et du comettage; $7^{\circ}$ enfin un chantier de rupture garni d'agrès, d'engins et de romaines, pour connaître avec précision la résistance des aussières et grelins ou petits câbles, de la circonférence de trois pouces jusqu'à trois pouces et demi.»

La manufacture de Popincourt dispose-t-elle de tous ces aménagements? On ne le sait. En revanche, on sait qu'elle comporte plusieurs ateliers «tant pour la fabrication du spart que pour les ouvrages en filasse» d'agave, et qu'elle occupe plus de quatre-vingt ouvriers dans les ateliers de la rue Popincourt, et plus de cent cinquante autres dans le faubourg Saint-Antoine et à Bicêtre (Monnier 1981), seulement pour tresser le spart, travail qui se fait à la main et requiert une main-d'œuvre peu qualifiée, donc peu rémunérée, mais nombreuse. 


\section{Une industrie prospère}

La manufacture produit des objets d'usage banal, comme des «cordages élastiques, qui n'ont pas, comme le chanvre, l'inconvénient de tacher les corps mouillés qu'on y étend, tels que le linge, le papier, les étoffes nouvellement teintes, qu'il s'agit de faire sécher», des nattes, des hamacs et des tapis «à peluches et sans peluches, propres aux salles à manger, bureaux, secrétaires, galeries, chambres, cabinets, aux équipages, etc. ».

Elle produit aussi «des nattes et des tapisseries susceptibles de faire ornement ${ }^{13}$ ». Gavoty développe des produits nouveaux, auxquels il donne un «degré de perfection, conforme aux besoins et au luxe de la capitale ${ }^{14} »$, destinés à une clientèle plus sophistiquée, qui s'inscrivent dans le développement du commerce de luxe à Paris.

Ainsi, au-delà des usages domestiques classiques (tapis pour pièces d'habitation ou églises, plafonds de voiture, tables de jeux; glands et cordons pour stores de voitures, sonnettes et lustres; cordes à puits, à linge; balais à palmes; cabas de jardinage, etc.), Gavoty joue sur l'engouement nouveau des classes sociales supérieures pour les jardins, les fausses grottes, etc., et développe des produits nouveaux qui retiennent l'attention d'une nouvelle clientèle. Dans les archives du Petit Trianon et dans celles des jardins de Méréville, on trouve mention d'achats de tapis imitant le gazon naturel et de fleurs artificielles en filasse d'aloès, provenant des ateliers de Gavoty ou de ceux de son successeur, Bonnet de Montprin. Un feuillet publicitaire, daté de 1787, énumère une production très diversifiée: «Tapis en corbeilles de parterre, ronds et ovales, variés de couleurs agréables et solides, pour cabinets, grottes et jardins, et pour les sallons, au bas des canapés [...]. Tapis imitant parfaitement le gazon naturel, fait par de nouveaux procédés de teintures, qui ont la propriété de conserver le spart sans lui laisser l'odeur désagréable dont on s'est plaint des autres fabrications. Tapis façon des Indes et de Hollande, pour tapisserie des anglaises et grottes de jardins ${ }^{15}$.» (Goueric 2006). Et les factures retrouvées font état de la livraison de pièces importantes à la fois en nombre et en superficie, près d'une centaine de pieds carrés.

Ce qui fait la force de la "Corderie nouvelle», c'est l'habileté de son directeur à élargir la gamme de ses productions pour qu'elles s'adressent à toutes les classes de la société:

«On voyait chez les riches des nattes et des tapis de toute couleur, des peluches imitant le gazon [...] des ouvrages d'agréministeries ${ }^{16}$ en aloès imitant la soie; et l'artisan employait des cordeaux, des cordes et des câbles de spart.»

Ces produits trouvent leur public et leur débit est assez considérable pour justifier l'existence, outre la manufacture de la rue Popincourt, de deux dépôts de marchandises à Paris et d'un dépôt de cordages à Rouen en 1776, et, en 1787, de trois dépôts à Paris et d'un autre à Versailles.

Devant le succès rencontré, Gavoty poursuit des recherches botaniques pour perfectionner ses productions et acclimater de nouvelles espèces en France; les textes parlent d'un jardin botanique établi et planté à grand frais et d'un «cabinet rare d'histoire naturelle ${ }^{17} »$. Il envisage, semble-t-il, d'introduire dans les départements du Midi la culture de «l'agave americana 


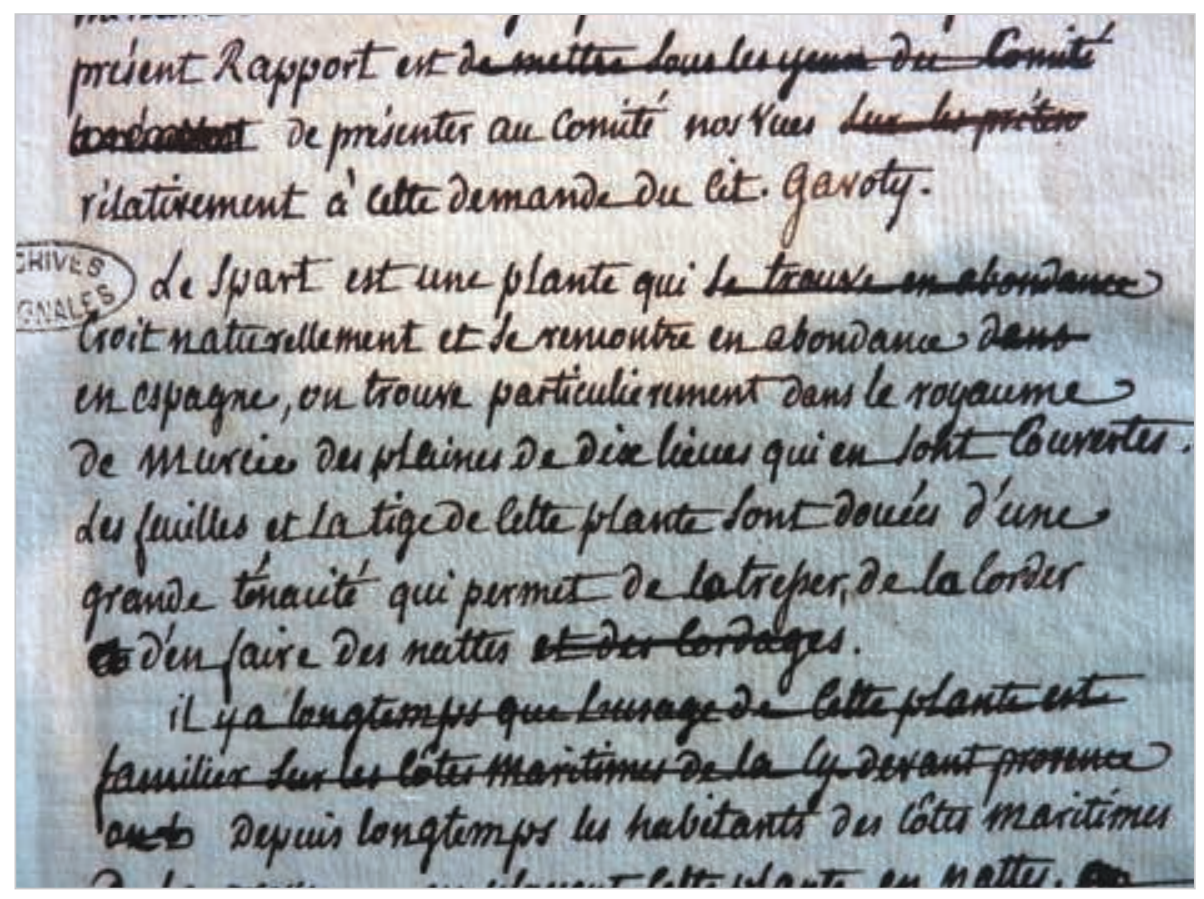

4. Origine et caractéristiques du spart et de ses usages Extrait d'un mémoire à la Commission d'agriculture et des arts, 7 fri maire an III, p.4, Archives nationales, $F / 12 / 2208$.

et foetida ${ }^{18}$, des yuca ${ }^{19}$, opuntia [ou cactus raquettes] et autres plantes propres à la filature et à la fabrication du papier; et, dans ceux de l'intérieur et du Nord, la culture du lygeum spartum, du spartum tertium et autres plantes filamenteuses $20 »$. Il travaille aussi à tirer de l'agave américaine « un papier, aussi beau que celui de Hollande et d'Angleterre, sans chiffons ordinaires, et seulement avec les étoupes formées de produits de diverses plantes qui me sont connues ${ }^{21} »$.

Il pousse ses recherches assez loin pour pouvoir produire au ministre de l'Intérieur, en l'an IV, un «in-folio contenant les échantillons d'ouvrages en spart, en agave d'Amérique et filasses extraites de divers végétaux propres à la filature et les étoupes utiles à la fabrication du papier ${ }^{22}$ ».

Une grande part de la réussite commerciale de Gavoty tient à son remarquable sens de la publicité. À l'intention de sa clientèle potentielle, il publie, dès novembre 1776, un «prospectus» de seize pages qui explique dans les détails ce qu'est le spart, les avantages que l'on peut tirer de son industrie, et qui fournit le catalogue très complet des produits de sa manufacture.

L'essentiel de ce prospectus est repris par les journaux, Gazette des arts et métiers, Gazette de santé, Mercure de France, qui répètent à l'envi les arguments de Gavoty: on rendra ainsi «au commerce des toiles la quantité de chanvre employée dans les corderies, [.. . et on] économisera la laine et la soie qu'il peut parfois remplacer ${ }^{23} »$.

La presse développe longuement les avantages du spart, particulièrement sur le plan de la salubrité, pour se préserver de l'humidité. Les hygiénistes recommandent son usage pour les nattes et tapisseries, de préférence aux peaux d'animaux et tapis de laine, peuplés de vermines et 
apportant des maladies, ou même au chanvre, réputé corrompre l'eau dans laquelle il séjourne. Le spart, lui, «se plaît dans l'humidité et résiste à ses effets, ce qui doit le faire préférer à tout autre moyen, surtout dans les salles à manger des rez-de-chaussée, dans les boutiques trop humides et trop fraîches ${ }^{24} \gg$.

De plus, tapisseries et tapis de spart peuvent se laver: «Ils gagnent au service, c'est-à-dire qu'ils deviennent plus beaux, plus fins et conséquemment plus chauds à mesure qu'on les lave et qu'on les peigne ${ }^{25}$.»

Le spart est donc promu comme un produit moderne, dans l'air du temps, qui répond aux préoccupations à la fois des hygiénistes et des Physiocrates, deux courants d'opinion influents en cette fin du XviII siècle; autant d'arguments à la mode, qui ne peuvent que séduire la clientèle.

Avec la même maîtrise de la communication qui règle ses relations avec la presse, Gavoty ouvre son entreprise aux visiteurs: «Les étrangers, les savants, et tout Paris visitèrent cette singulière manufacture. » La sparterie ou « Corderie nouvelle» de Popincourt est signalée dans des guides touristiques à l'usage des voyageurs, avec la description des produits qui sortent de ses ateliers: «Le tout y est solide, propre et à un prix honnête. » (Hurtaut \& Magny 1779).

5. Perfectionnement des procédés.

Extrait d'un mémoire à la Commission d'agriculture et des arts, 7 frimaire an III, p. 5 , Archives nationales, F/12/2208.

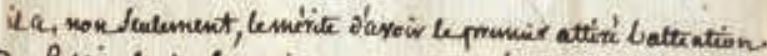
Du Pabic Jurla fpartivio : main encon den avoirfurfutionne

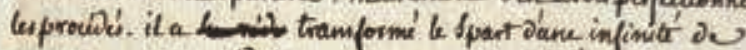

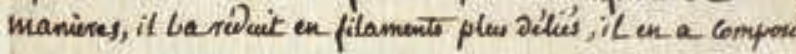

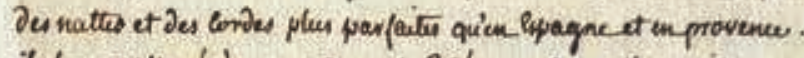
it La appliqué à un grain nombrd d'urages donustiques, is en as fabriqué Des tapis propres à dupléer lux de laine, Ju nattu

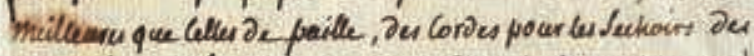

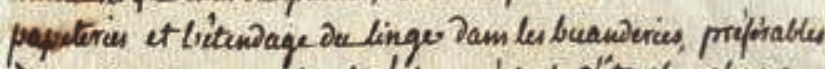
a Celler de chanve qui ont l'inconvinient J'ita ples cheres et De tacker, in leotrumpant, linge ot le Papier. de forr

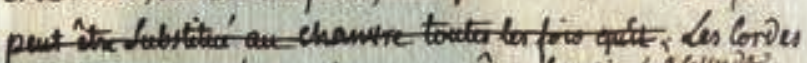

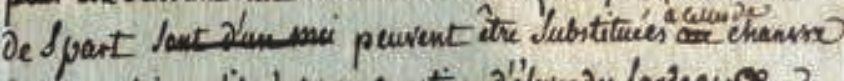
touter les foi quit nest pas question. J'eleverdes farieneuse? Coniwétables ou or vaiure de Grandes rivistanus, elles Falent:

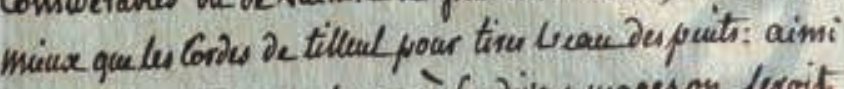
en appliquant cette prlante à Cu bivers uages on feroit 


\section{Revers de fortune}

Toutefois la prospérité ne dure pas. La guerre avec l'Angleterre de 1778 à 1783 porte un coup à cette industrie, car les relations maritimes avec l'Espagne sont menacées par les corsaires. Gavoty perd des marchandises, doit réinvestir des sommes assez importantes (il parle de 250000 livres), sans réussir à obtenir le soutien de Necker pour développer la culture du spart en France.

Malgré ces difficultés, il cherche à conforter son industrie et pousse plus avant ses recherches pour améliorer encore la qualité de ses produits. En $1780^{26}$, à nouveau, l'Académie des sciences est saisie d'expériences comparatives nouvelles faites avec des cordages de spart produits par la manufacture de Gavoty et des cordages de chanvre produits par un autre cordier parisien; elle en conclut que «les cordages de spart sont capables d'une grande résistance [mais] que leur force est toujours inférieure à celle des cordages de chanvre». L'Académie préconise d'employer le chanvre pour des cordages devant élever des fardeaux considérables ou devant travailler longtemps pendant la sécheresse, «mais, dans plusieurs autres cas, les cordages de spart peuvent être d'un très bon usage», par exemple, pour tirer l'eau des puits.

À la suite de démêlés avec le Mont de Piété qui lui a accordé un prêt moyennant le dépôt en garantie d'un chargement de sparterie, Gavoty est menacé d'une lettre de cachet qui l'oblige, en 1784, à se retirer en Angleterre 27. Pendant son absence, si on l'en croit, ses associés, Marguerite Lenoir, veuve Cogorde, et Bonnet de Montprin, «s'empare[nt] secrètement de [ses] biens et des fruits de [ses] travaux s'élevant à 400000 francs $^{28} »$. À son retour à Paris, en septembre 1785 , il dit découvrir la situation suivante:

«Si j'y trouvais mes commis, mes domestiques, le chef de mes ateliers et ouvriers, je n'y vis plus mes meubles, titres et papiers, plantations, machines; enfin, je trouvai ma manufacture de sparterie dans le plus grand désordre.»

Gavoty est finalement exclu de sa propre manufacture. Peut-être, plus prosaïquement, a-t-il mal géré ses affaires, comme le suggère l'administration du Commerce ${ }^{29}$, et s'est-il endetté visà-vis d'associés qui ont cherché à se rembourser.

Son successeur, Bonnet de Montprin, déjà fournisseur de la reine et des princes, obtient du roi la permission de fabriquer et vendre à Paris et ses environs des ouvrages de sparterie; mais, contrairement à ce qu'il espérait, il n'obtient ni privilège de fabrication, ni exemption des droits d'entrée sur le spart, ni le titre de manufacture royale ${ }^{30}$. Ensuite, il développera la fabrication, à base de fibres d'aloès, d'étoffes imitant le marli et la gaze, propres à faire des chapeaux pour femmes, toujours sans privilège exclusif.

La manufacture de la rue Popincourt ne survit pas à la Révolution. Elle est au centre d'une âpre bataille judiciaire entre Gavoty et ses «spoliateurs » qui traîne en longueur, se complique du fait que les anciennes juridictions sont supprimées par la Révolution, et surtout qui épuise les ressources de Gavoty qui va connaître de bien sombres années.

Un temps, il fonde de grands espoirs, qui seront déçus, sur la législation protectrice de l'invention votée en 1790-1791, et milite à la Société des inventions et découvertes (Demeulenaere-Douyère 


\section{Usage des diverses plantes.}

Spart, agave, extrait d'un mémoire à la Commission d'agriculture et des arts, 7 frimaire an III, p.5, Archives nationales, F/12/2208.

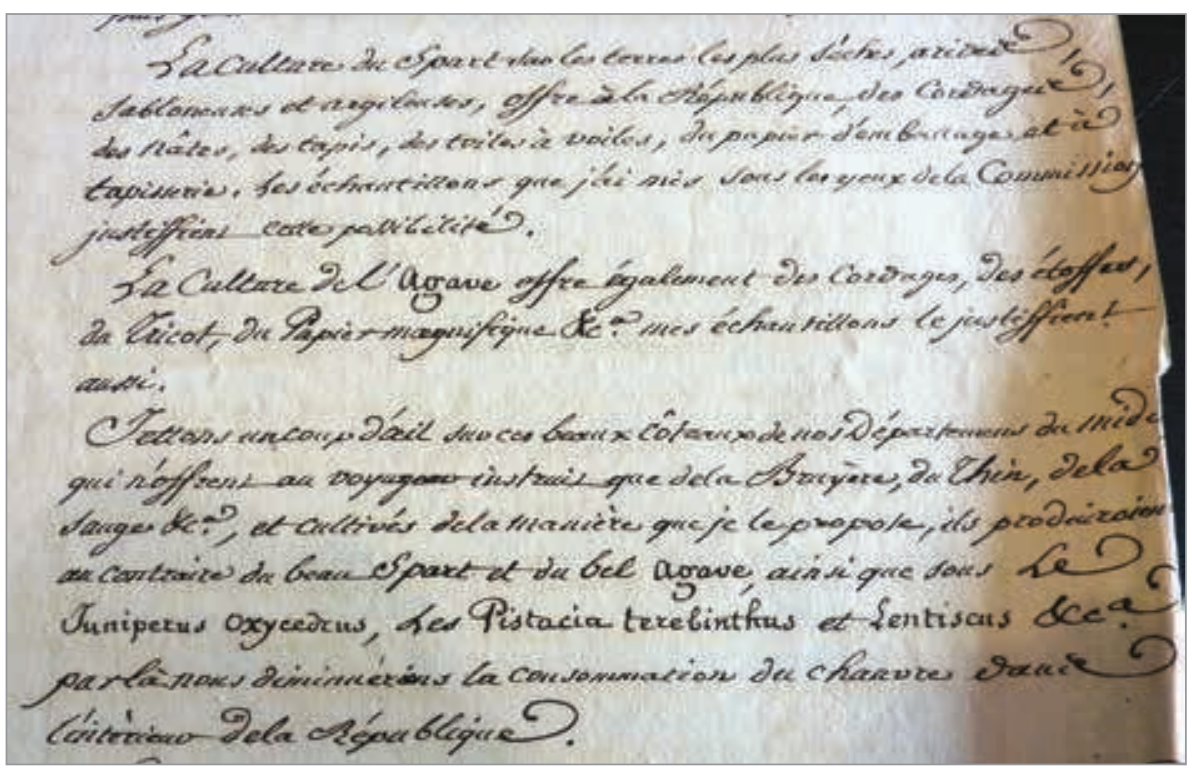

2009), en vain. Se posant en victime des «manœuvres de l'ancien et tyrannique régime ${ }^{31} »$, il entretient avec les instances officielles un long et difficile dialogue au cours duquel il tente de faire valoir ses compétences et d'obtenir dédommagement, soutien et subventions ${ }^{32}$. Se détournant du spart, Gavoty consacrera ses dernières années à tenter de perfectionner l'art de la corderie en se fondant sur une meilleure connaissance de la constitution du chanvre.

Jean-François Gavoty meurt à Paris, âgé de près de 80 ans, le 7 décembre 1812, seul, dans un misérable garni de la rue de la Montagne Sainte-Geneviève. Quand on dresse l'inventaire de ses biens, le 29 août 1813, on constate qu'il n'a laissé qu'une caisse contenant quelques hardes hors d'usage et « cinq cent volumes brochés in- $8^{\circ}$ de l'Imprimerie de Pelletier ayant pour titre Manuel du fileur cordier, [...] prisé vingt-cinq francs, avec deux cartons renfermant des projets, mémoires, notes et autres pièces relatives à l'ouvrage ci-dessus inventorié 33 ».

La prisée de ces ouvrages qui sont le fruit laborieux de ses derniers combats ne couvre pas le montant des loyers dus à son logeur.

Quant à la sparterie, malgré ses efforts pour jouer sur l'effet de mode et s'adresser à de nouveaux publics et malgré aussi un succès attesté, au moins dans certains secteurs de la société, on peut s'interroger sur la réelle diffusion des produits de sa «Corderie nouvelle». Dans les inventaires après décès qu'elle a étudiés, Annik Pardailhé-Galabrun (1988) n’a pas trouvé de tapis ou d'objets en sparterie, seulement des nattes de jonc, qu'elle qualifie de « revêtement rare et original», chez un maître boulanger. Mais il est vrai qu'il s'agit là de produits fragiles, peu pérennes, de faible prix, peu prestigieux, qu'on n'est guère enclin à conserver et que les notaires peuvent négliger de consigner dans les inventaires après décès. 
Le cas de la sparterie, que Gavoty s'attache à promouvoir avec opiniâtreté et talent, illustre quelle réhabilitation une matière première qui est moins un « reste» qu'un laissé pour compte, un matériau jusque-là délaissé et méprisé, peut trouver, surtout si le savoir-faire de l'entrepreneur, la mode et l'engouement du public se combinent pour en faire un produit de luxe prisé.

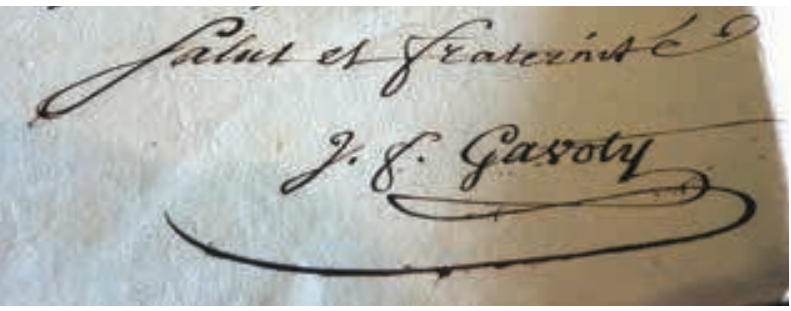

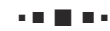

\section{Notes}

1. On se reportera notamment aux travaux du Centre d'étude des techniques et de recherche expérimentale en préhistoire (CETREP) (www.chercheursdelawallonie.be/cetrep_sparterie.html) qui a reconstitué des objets en sparterie.

2. «Spart», dans C. Meyer (éd.), Dictionnaire des sciences animales, Montpellier, Cirad, 2012 (dicosciences-animales.cirad.fr/liste-mots.php?fiche $=25561 \&$ def $=$ spart $)$; Dictionnaire de français Littré (littre.reverso.net/dictionnaire-francais/ definition/sparte/69891).

3. À la fin du xixe siècle, on tirera même de l'alfa d'Algérie une pâte à papier recherchée.

4. On trouve des exemples de la production actuelle d'objets en sparterie, particulièrement en Espagne, sur pasperdus.canalblog.com/ archives/2008/02/16/7891143.html.

5. On peut faire le parallèle à cet égard avec les efforts de l'« architecte rural » François Cointeraux (17401830), contemporain de Gavoty, pour promouvoir un nouvel usage du pisé, particulièrement dans la construction des bâtiments agricoles; à ce sujet voir les références citées.

6. Né le 13 août 1733, à Toulon, paroisse Sainte-Marie, il est l'un des 13 enfants de Joseph-François Gavoty (1687-1770) et de Thérèse Solliers; son plus jeune frère, Jean-Baptiste Charles Gavoty (1748-1795), capitaine de vaisseau (16 ans de marine marchande en Chine, Inde et Égypte) émigrera chez les Anglais en 1793 à la prise de Toulon.

7. Un «Gavoty, négociant» figure parmi les députés élus par le Tiers-État de Toulon le 17 mars 1789, Gustave Lambert, Histoire de Toulon, partie 2, t. 4, [Toulon], impr. du Var, 1886-1892, p. 444.

8. Archives nationales [ensuite AN], E/1519/A, fol. 1 .

9. La première entreprise à laquelle Gavoty participe, après son départ de Toulon, est, en 1772, le dessèchement de marais dans la région de Dunkerque.

10. Archives de l'Académie des sciences, Paris, procès-verbaux, séance du 2 août 1775 .

11. AN, E/1519/A, fol. 1-2, 3 octobre 1775.

12. Un exemplaire dans AN, F/12/2208.

13. Mercure de France, septembre 1776.

14. Gazette des arts et métiers, 17 août 1775.

15. Nicole Goueric qui cite les recherches de Gabriela Lamy sur le jardinier Antoine Richard (note 47); citation, p. 22.

16. L'agréministe est l'ouvrier passementier dont le travail spécial s'applique aux ornements de la parure des femmes, à ceux des meubles, etc. (Trésor de la langue française informatisé, dvlf.uchicago.edu/mot/ agre\% CC\%81ministe).
7. La signature de Jean-François Gavoty. An III, Archives nationales, F/12/2208 
17. AN, AD/XVIII/c/312/nº 53, Gavoty 1791a: 10 et 20

18. À la Réunion, le choca (ou aloès vert, urcraea foetida) a été longtemps cultivé pour la production d'une fibre textile utilisée dans la fabrication de cordages; on utilise encore ses feuilles découpées en lanières, en guise de ficelles, et la moelle de ses hampes florales sert dans l'artisanat local; on utilise aussi son cœur dans la cuisine locale.

19. Yucca: plante originaire d'Amérique du Nord et centrale, comprenant une grande variété d'espèces, dont on tire des fibres très résistantes (type sisal), qui sont utilisées à la fabrication de cordage, de ficelle, de tissus grossiers et de tapis. Sur le traitement de ces fibres: www.primitiveways.com/yucca_processing.html; sur d'éventuelles utilisations: paleotool. com/2011/11/30/yucca-fiber-skirt/.

20. AN, F/12/2208, lettre de Gavoty au ministre de l'Intérieur, $1^{\text {er }}$ messidor an IV [19 juin 1796].

21. Ibid., lettre de Gavoty au ministre de l'Intérieur, 15 prairial an IV [3 juin 1796].

22. Ibid., «Inventaire des pièces qui accompagnent ma pétition adressée au Citoyen ministre de l'Intérieur du 15 prairial an IV ».

23. Mercure de France, septembre 1776, p. 191-193.

24. Gazette de santé 35, 29 août 1776 .
25. Mercure de France, op. cit.

26. Archives de l'Académie des sciences, Paris, pochette de la séance du 2 sept. 1780 ; expériences commentées dans le Supplément au $n^{\circ} 298$ du Journal de Paris, 24 oct. 1780

27. Il y travaille à perfectionner une machine hydraulique de son invention dont il prétendra plus tard, sous la République, qu'elle connut un vif succès, mais qu'il préféra en réserver le bénéfice à ses compatriotes, Gavoty 1791a.

28. Gavoty 1791b.

29. AN, F/12/707, p. 320, 10 juillet 1788: Tholozan indique que Gavoty a vendu son établissement à Bonnet de Montprin, à qui il refuse le privilège exclusif de vingt années demandé pour les ouvrages de sparterie qu'il fabrique.

30. AN, E/1636/A, 13 septembre 1785.

31. AN, F/12/2208, lettre de Gavoty à la Commission d'agriculture et des arts, 6 brumaire an III [27 octobre 1794].

32. En avril 1792, Gavoty obtient du Bureau de consultation des arts et métiers une récompense de première classe de 6000 livres, soit le maximum.

33. AN, ET/XLII/756, inventaire après le décès de Jean-François Gavoty, 29 août 1813

\section{I'auteure}

Christiane Demeulenaere-Douyère est docteure en histoire et conservatrice générale du patrimoine, membre correspondant du Centre Alexandre Koyré, UMR 8560 / CNRS-EHESS-MNHN (Paris). Ses recherches portent sur l'histoire institutionnelle, sociale et culturelle de l'innovation technique.

\section{Iconographie}

Image d'ouverture. Alfa ou stipa tenacissima (ici au Portugal) (C) Carsten Niehaus.

1. C) CETREP, 2005.

2. fr.topic-topos.com/objets-de-la-sparterie-dammartin -en-serve.

\section{Références}

Archives de l'Académie des sciences. Paris: procès-verbaux, séance du 2 août 1775 ; pochette de la séance du 2 septembre 1780 .

Archives nationales: AD/XVIII/c/312/nº 53; E/1519/A, fol. 1-2, 3 octobre 1775; E/1636/A, 13 septembre
3. (C) B. Cros.

4, 5, 6 et 7. (C) C. Demeulenaere-Douyère.

8. journees-du-patrimoine.com/SITE/musee-anciennesparterie-mas--damma-40446.htm.

1785; F/12/707, p. 320, 10 juillet 1788; F/12/2208; ET/XLII/756, 29 août 1813.

Baridon, L., Garric, J.-P. \&J.-P. Richaud dir. 2016 Les Leçons de la terre. François Cointeraux (1740-1830), professeur d'architecture rurale. Paris: INHA/Éditions des cendres. 
Demeulenaere-Douyère, C. 2016 «François Cointeraux (1740-1830) à Paris. Le temps des "écoles d'architecture rurale" », in F. Fleury, L. Baridon, A. Mastrorilli, R. Mouterde \& N. Reveyron dir., Les temps de la construction. Processus, acteurs, matériaux, Recueil de textes issus du $2^{e}$ congrès francophone d'histoire de la construction, Lyon, 29-31 janvier 2014. Paris: Éditions Picard: 1067-1077.

- 2009 «Inventeurs en Révolution: la Société des inventions et découvertes», Documents pour l'histoire des techniques $17,1^{\text {er }}$ sem. : 19-56.

Garin, J. 1910 «Les Annonciades de Popincourt (16361782). Leur établissement, les deux premières supérieures », Revue d'histoire de l'Église de France 1: 533-554 et $666-681$, et $2: 17-23$

Gavoty, J.-F. 1810 Art de la corderie. Sur la constitution du chanvre et le salut du navigateur. Paris: Imprimerie de Pelletier: 7-8.

- 1792 Mémoire à Messieurs les Députés à l’Assemblée nationale, ci-devant de Berthe, créateur et propriétaire de la manufacture de sparterie... Paris: Imprimerie du Patriote français, s. d. [1 ${ }^{\text {er }}$ janvier 1792] : 3 .

- 179la Réclamation de propriété industrielle. Paris: Imprimerie du Patriote français: 10, 20.

- 1791b Mémoire à MM. les Juges du IIIe tribunal séant au Chatelet, Paris: Imprimerie du Patriote français.
- 1776 Manufacture de sparterie autorisées par arrêt du Conseil d'Etat du roi du premier octobre 1775, établie à Paris rue Popincourt faubourg Saint-Antoine, Paris: Imprimerie de B. Morin.

Gazette des arts et métiers, 17 août 1775.

Goueric, N. 2006 «Histoires de brins et brin d'histoire. Ruminations sur gazons et faux gazons dans les jardins du xvine siècle », Polia 5 : 7-30.

Hurtaut et Magny, 1779 Dictionnaire historique de la ville de Paris et de ses environs, t. 4. Paris: Moutard: 662-663.

Journal de Paris, 24 octobre 1780, Supplément au n² 298.

Lambert, G. 1886-1992 Histoire de Toulon, partie 2, t. 4, Toulon: Imprimerie du Var: 444.

Lunier, 1805 Dictionnaire des sciences et des arts, contenant l'étymologie, la définition et les diverses acceptions des termes techniques..., t. 3, Paris: Gide et Nicolle: 354.

Monnier, R. 1981 Le Faubourg Saint-Antoine (1789-1815), Paris: Société des études robespierristes: 63.

Pardailhé-Galabrun, A. 1988 La Naissance de l'intime. 3000 foyers parisiens XVII -XVIII ${ }^{e}$ siècle. Paris: PUF : 373.

Rougier de La Bergerie, J.-B. 1800 Mémoire sur la culture, le commerce et l'emploi des chanvres et lins en France pour la marine et les arts. Paris: 16-17.

Thillay, A. 2002 Le Faubourg Saint-Antoine et ses «faux ouvriers ». La liberté du travail à Paris aux XVII et XviIle siècles. Paris: Champ Vallon.

\section{Pour citer cet article}

Demeulenaere-Douyère, C. 2016 «La sparterie selon Jean-François Gavoty. D’un matériau "pauvre" à un produit de luxe», TechniquesE Culture 65-66 «Réparer le monde. Excès, reste et innovation », p. 180-193.

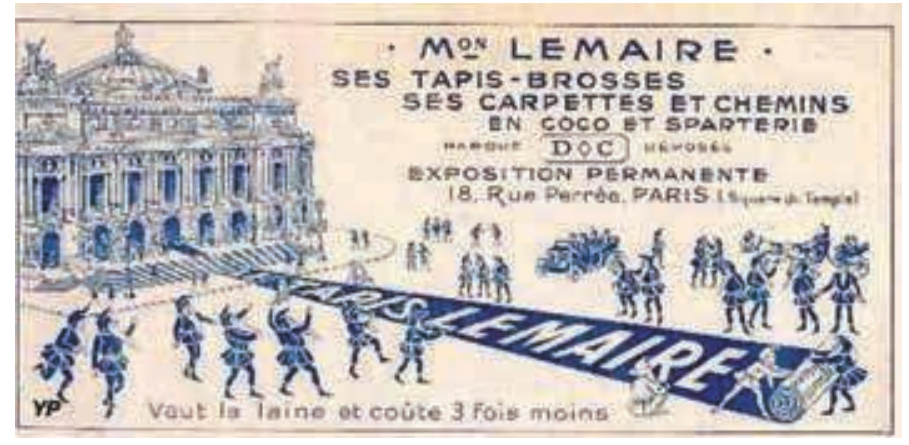

8. Publicité pour la «Maison Lemaire, tapis-brosses, carpettes et chemins en coco et sparterie ».

Musée de l'Ancienne Sparterie - Le MAS, Dammartin-en-Serve (78). 